https://helda.helsinki.fi

\title{
Annual and diurnal water vapor cycles at Curiosity from observations and column modeling
}

\section{Savijärvi, Hannu}

2019-02

Savijärvi , H , McConnochie , T H , Harri , A-M \& Paton , M 2019 , ' Annual and diurnal water vapor cycles at Curiosity from observations and column modeling ' , Icarus, vol. 319 , pp. 485-490 . https://doi.org/10.1016/j.icarus.2018.10.008

http://hdl.handle.net/10138/320371

https://doi.org/10.1016/j.icarus.2018.10.008

cc_by_nc_nd

acceptedVersion

Downloaded from Helda, University of Helsinki institutional repository.

This is an electronic reprint of the original article.

This reprint may differ from the original in pagination and typographic detail.

Please cite the original version. 


\title{
Annual and diurnal water vapor cycles at Curiosity from observations and column modeling
}

\author{
Hannu Savijärvi ${ }^{1,2, *}$, Timothy H. McConnochie ${ }^{3,4}$, Ari-Matti Harri ${ }^{2}$, Mark Paton ${ }^{2}$
}

${ }^{1}$ Institute for Atmospheric and Earth System Research / Physics, University of Helsinki, Finland ${ }^{2}$ Finnish Meteorological Institute, Helsinki, Finland

${ }^{3}$ Department of Astronomy, University of Maryland, College Park, MD20742, United States

${ }^{4}$ NAS A Goddard Space Flight Center, Greenbelt, MD20771, United States

revised 5.10.2018

\begin{abstract}
Local column precipitable water contents (PWC) for more than a martian year from 113 Curiosity ChemCam passive-mode sky scans were used to force a column model with subsurface adsorption. ChemCam volume mixing ratios (vmr) and T, RH and vmr from REMS-H were compared with model results. The REMS-H observations point to decrease of vmr (i.e. depletion of near-surface water vapor) during every evening and night throughout the year. The model's pre-dawn results are quite similar to the REMS-H observations, if adsorption is allowed. The indicated porosity is about $30 \%$ and the night depletion ratio about 0.25 . If adsorption is not allowed, $\mathrm{RH}$ and vmr become excessive during every night at all seasons, leading to ground frost between $\mathrm{Ls} 82^{\circ}-146^{\circ}$; frost has not been observed. As brine formation is unlikely along the Curiosity track, adsorption thus appears to be the depleting process.

During daytime the ChemCam vmr is in general close to surface values from the Mars Climate Database (MCD) vmr profiles for the Curiosity site when those profiles are scaled to match the ChemCam PWC. Our simulated daytime surface-vmr is in turn close to the ChemCam vmr when moisture is assumed well-mixed to high altitudes, whereas a low moist layer $(15 \mathrm{~km})$ leads to overestimates, which are worse during the warm season. Increased TES-like regional PWC also leads to large overestimates of daytime surface-vmr. Hence the crater appears to be drier than the region surrounding Gale and the results support a seasonally varying vertical distribution of moisture with a dry lower atmosphere (by Hadley circulation), as suggested by MCD and other GCM experiments.
\end{abstract}

Keywords: Mars, climate Mars, surface Meteorology

*Corresponding author at: INAR/Physics, Faculty of Science, 00014 University of Helsinki, Finland

E-mail address: hannu.savijarvi@helsinki.fi

Tel: $+358-40-9380858$ 


\section{Introduction}

The martian water cycle has been the object of intense research through orbit observations and general circulation model experiments (GCM), reviewed recently by Montmessin et al. (2017). Martinez et al. (2017) reviewed all surface-based observations. Among the less well-known aspects of the water cycle are the surface interactions and the vertical distribution of moisture, both being difficult to observe. This article is an attempt to study them via the Mars Science Laboratory (MSL) observations onboard the Curiosity rover in the Gale crater. We use the ChemCam passive-mode sky scans for column precipitable water content (PWC, McConnochie et al., 2018) and the Rover Environmental Monitoring Station humidity measurements (REMS-H, Harri et al., 2014a, GomezElvira et al., 2012) during more than one martian year (MY). A column model is applied to help interpret the observations.

According to GCM experiments and data assimilations (Richardson and Wilson, 2002; Navarro et al., 2014; Steele et al., 2014; Mars Climate Database (MCD, Millour et al., 2015); Montmessin et al., 2017) the equatorial latitude of Gale $\left(4.6^{\circ} \mathrm{S}\right)$ is relatively dry near the surface throughout most of the martian year, because of transport by the large-scale Hadley circulation. High mixing ratios of water vapor would hence be expected aloft, at 10-15 km during the cool aphelion season (Ls $\sim 71^{\circ}$ ) and at 30-40 km during the warm perihelion season $\left(\mathrm{Ls} \sim 251^{\circ}\right)$, but these model predictions have been hard to prove by observations. The moist season of high PWC would occur at Gale at around Ls $180^{\circ}$, when the water vapor pulse from the sublimated north polar ice cap finally reaches the equatorial latitudes.

At the surface, the possible exchange of water with the surface has been debated since the Viking observations, which according to Jakosky et al. (1997) displayed depletion of near-surface water vapor each night that was not due to formation of frost. They suggested and modeled regolith adsorption as the reason. Evening depletion has been reported in the TECP data of Phoenix (Zent et al., 2016) and depletion is present in REMS-H data (Harri et al., 2014a, Savijärvi et al., 2015). Surface brine formation might also deplete air moisture (Martin-Torres et al., 2015) but this is not favored by Curiosity data (Rivera-Valentin et al., 2018). Savijärvi et al. (2016, 2017) used a column model with adsorption and frost to explain the first-sols observations of Viking and Curiosity. Steele et al. (2017) applied an adsorptive mesoscale model for the Gale region but they, too, considered just a few sols, although during the three main seasons.

Here observations and the column model with and without adsorption are used to study the diurnal and annual cycles of water vapor at Curiosity. The model is forced by the rover's strictly local ChemCam daytime PWC through more than one martian year, and the REMS-H observations and model results for diurnal temperatures, relative humidities and mixing ratios are compared at the observation height of $1.6 \mathrm{~m}$. Two vertical profiles for the model's initial mixing ratio are tested. Furthermore, increased values of PWC, closer to the regional orbit retrievals by TES and CRISM over Gale, are tried for comparison.

Observations and model experiments are described in Section 2. Section 3 demonstrates the diurnal cycles during the warm season, and Section 4 respectively during the cool season (the moist season was discussed in Savijärvi et al., 2016). The full annual cycle is then considered in Section 5 and the findings are discussed in Section 6. Conclusions are given Section 7.

\section{Observations and model experiments}

The MSL ChemCam passive spectroscopic scans of scattered skylight can be used to provide local estimates of column precipitable water content (PWC) and the dust and water ice fractions of the 
total $880 \mathrm{~nm}$ aerosol optical depth $\tau$ from MastCam, given the surface pressure $\mathrm{p}$, which is measured by the MSL REMS-P device (Harri et al., 2014b). The methodology and first results are described in McConnochie et al. (2018). We use their results, which originate from $113 \mathrm{ChemCam}$ passive sky scans from MSL sols 230-1293 (Ls 291 ${ }^{\circ}$ MY31 to Ls $127^{\circ}$ MY33). The scans are mostly from daytime, between 10-13 Mars hours local mean solar time (LT); some are from the morning, 07-09LT. The indicated precision for PWC is +-0.6 $\mu \mathrm{m}$. We assume in the following that the ChemCam values represent the strictly local conditions along the Curiosity track on the Gale crater base.

The simultaneous measurements of PWC, $\tau$ (mostly due to dust) and $p$ are shown in Figure 1 as function of season. Since $\tau$ and $p$ are not originally simultaneous with the ChemCam PWC scans, we apply the same interpolation procedure that was used by McConnochie et al. (2018). One may note low PWC and dustiness during the cool aphelion - southern late fall season at around Ls $71^{\circ}$ $90^{\circ}$, a moist season with high PWC and low $\mathrm{p}$ at around Ls $180^{\circ}$, and a long dusty mid-PWC period during the warm perihelion - southern late spring, $\operatorname{Ls} 251^{\circ}-270^{\circ}$. The few morning values are similar to the surrounding daytime PWC retrievals, indicating little or no diurnal variation of PWC.

These local ChemCam PWC retrievals, when scaled to the traditional reference p of $6.1 \mathrm{mb}$, appear consistently lower by 2-4 $\mu \mathrm{m}$ than the similarly-scaled regionally averaged retrievals from TES and CRISM in McConnochie et al. (2018). The large averaging area for the orbital data sets spanned 40 degrees of longitude and 10 degrees of latitude. When CRISM averaging was restricted to a local scale covering only the interior of the Gale crater (Toigo et al., 2013), the resulting lower PWCs become consistent with ChemCam. This suggests that low daytime PWCs are a real feature of the crater rather than some kind of instrumental bias. In the Steele et al. (2017) high-resolution simulations daytime upslope winds flush the desorbed moisture out of the crater base (cf. their figs. $15 \mathrm{~h}, 16 \mathrm{f}$ ), thus keeping the crater relatively dry during all the three seasons they considered.

The MSL REMS-H device onboard Curiosity consists of a fast temperature sensor and three relative humidity Vaisala sensors in a dust-protected cage $1.6 \mathrm{~m}$ above the surface (Harri et al. 2014a). We use here the hourly $5 \mathrm{~min}$ averages of $\mathrm{T}$ and the first hourly measurements of RH of selected sols (data from the Planetary Data System). The value for the volume mixing ratio of water vapor at 1.6 $\mathrm{m}(\mathrm{vmr})$ is calculated from the observed $\mathrm{p}, \mathrm{T}$ and $\mathrm{RH}\left(\mathrm{vmr}=\mathrm{RH} \cdot \mathrm{e}_{\mathrm{sat}}(\mathrm{T}) / \mathrm{p} ; \mathrm{e}_{\text {sat }}\right.$ is the saturation pressure of water vapor with respect to ice) as in Savijärvi et al. (2016). Because the cage is wellventilated, the REMS-H temperatures closely follow the free air temperature at $1.6 \mathrm{~m}$. Values for $\mathrm{RH}$ and vmr are most accurate at maximum RH (minimum vmr). This typically occurs daily during nighttime, at 04-06LT (cf. Figures 3-4). During daytime RH is very small $(<2 \%)$ and the REMS-H -derived values for vmr unfortunately become unreliable.

The column model is the same as used for MSL and described in detail in Savijärvi et al. (2016) with two small refinements explained below. The atmospheric part is hydrostatic with constant geostrophic wind of $10 \mathrm{~m} / \mathrm{s}$ (leading to near-surface winds of about $2 \mathrm{~m} / \mathrm{s}$ at nighttime and about 5 $\mathrm{m} / \mathrm{s}$ during daytime). There are 29 levels from $0.3,0.7,1.6,7 \mathrm{~m} \ldots$ to $50 \mathrm{~km}$. Parameterizations include radiation, turbulence and interactive cloud physics. Subsurface vertical diffusion of soil temperature and pore volume moisture with possible adsorption of moisture on regolith grains is represented in eight levels from the surface down to $48 \mathrm{~cm}$ depth. Adsorption isotherm follows the Jakosky et al. (1997; J97) formulation. Icefog, iceclouds, surface frost and pore ice form during supersaturation and sublimate under subsaturation with the associated latent heat and radiative effects.

The two refinements to the Savijärvi et al. (2016) regolith scheme are as follows:

1) The coefficient $\mathrm{D}$ for molecular/Knudsen diffusion of water vapor in $\mathrm{CO}_{2}$ pore volume gas, 
which was $1 \mathrm{~cm}^{2} / \mathrm{s}$, is increased to $5 \mathrm{~cm}^{2} / \mathrm{s}$, based on test chamber experiments in martian pressures, temperatures and regolith representations of Hudson et al. (2007). The diurnal minima and maxima of vmr do not change by this but the late morning and early evening behavior is closer to the recalibrated Phoenix TECP results of Fischer et al. (2018).

2) The scaled diffusion coefficient during active adsorption $D^{\prime}$ is no longer constant but is allowed to vary with the predicted regolith temperature and pore space moisture at each depth. The effect of this is small as $\mathrm{D}^{\prime}$ and its variations with temperature and moisture are quite small, $\mathrm{D}^{\prime} \sim 3 \cdot 10^{-6} \mathrm{~cm}^{2} / \mathrm{s}$.

In the present Curiosity simulations thermal inertia is 300 SI units, surface albedo 0.18 , surface roughness length $1 \mathrm{~cm}$ and regolith porosity 0.3 for simplicity, even though these may vary in reality along the track. The model is initialized at 00LT for each Ls of Figure 1 from the observed PWC, $\tau$ and $\mathrm{p}$ (with dust well-mixed and T $210 \mathrm{~K}$ at the surface, lapsing $1 \mathrm{~K} / \mathrm{km}$ ). It is then run to sol three with $\mathrm{p}$ and $\tau$ constant, by which time its winds, temperatures and mixing ratios have spun up at all heights to a repeating diurnal cycle, which closely preserves the initial PWC (for porosity of 0.3 ). The model values shown are from this last sol.

Initialization of the unknown moisture profile to the ChemCam PWC is made using two simple alternatives. The first is a high-layered well-mixed assumption ("well-mixed"), in which the water vapor mixing ratio is the same at all heights up to $50 \mathrm{~km}$. The other assumes a $15 \mathrm{~km}$ high wellmixed layer, above which vmr decays to zero ("low moist layer"). This is based on GCM coolseason results (e.g. Montmessin et al., 2017, fig. 11.18), in which moisture at the equatorial latitudes appears concentrated nearer the surface, maximum vmr being typically at around 10-15 km height with a rapid decay aloft. To obtain the same ChemCam PWC, vmr must now be about $26 \%$ higher in this low layer than in the high layer case. The well-mixed assumption might be expected to be better during the warm season and the low moist layer assumption during the cool season. The pore volume mixing ratio is initialized to the boundary layer mean $(\sim 4 \mathrm{~km})$ of the initial air mixing ratio in each experiment. Cloud processes are switched off above $7 \mathrm{~km}$ (the well-mixed profile would lead to extreme cool season supersaturations at high altitudes), but fogs and boundary layer clouds are allowed to occur as in the Viking and Phoenix simulations of Savijärvi et al. (2010, 2017). However, they do not occur in any of the present integrations, due to the fairly dry equatorial Gale environment.

Two further experiments are made using the well-mixed initial moisture. As the strictly local ChemCam results for PWC appear consistently 2-4 $\mu \mathrm{m}$ lower than the large-scale regional TES and CRISM retrievals (McConnochie et al., 2018), we trace the sensitivity of our results to this by increasing all the ChemCam PWC values by $4 \mu \mathrm{m}$. In a fourth experiment adsorption is set to zero, but vertical diffusion of moisture remains active in the porous regolith. There are thus four simulations for each PWC observation of Figure 1: well-mixed ("wm"), low moisture layer ("low m"), increased moisture ("high pwc"), and no adsorption ("no ads").

Figure 2 displays for later reference the model's $1.6 \mathrm{~m}$ temperatures at $05 \mathrm{LT}$ and at the scan hour of each observation of Figure 1. The model temperatures are practically the same in all four experiments and are quite close to the respective REMS-H observations (Figures 3-4). Figure 2 indicates that the morning ChemCam scans are mostly from around 08LT (with one at 09:00LT and one at 07:15LT). The 102 daytime observations are mostly from around 11LT and 12LT, with a few around 10LT and 13LT. The cool and warm seasons are clearly visible in Figure 2 and the moist season in Figure 1.

\section{Diurnal comparison during the warm season}


First comparisons between the REMS-H observations and the adsorptive column model results were made in Savijärvi et al. (2016) for MSL sols 15-17 and 80-82 during the moist season. The model was initialized at $\mathrm{L}_{\mathrm{s}} 159^{\circ}$ and $196^{\circ}$ with an approximate PWC of $9.3 \mu \mathrm{m}$ and $8 \mu \mathrm{m}$, respectively. Results indicated good matches for diurnal temperatures and relative humidities at 1.6 $\mathrm{m}$. A fair match with the REMS-H mixing ratios was obtained during the early morning hours. In this section the observed and modeled diurnal cycles are similarly compared during the warm season.

Figure 3 displays hourly REMS-H observations for three sols at around Ls $271^{\circ}$ and the model's diurnal cycle arising from ChemCam MY32 Ls $270.96^{\circ}$ 10:20LT scan, PWC being $7.45 \mu \mathrm{m}, \tau 0.87$ with dust fraction 0.83 , and p $9.20 \mathrm{mb}$. The observed $\mathrm{T}$ (top panel) ranges between $205-273 \mathrm{~K}$. The model's $\mathrm{T}$ at $1.6 \mathrm{~m}$ is quite close to the observations. Model's surface temperature $\mathrm{T}_{\mathrm{s}}$ is also displayed.

The observed relative humidities (mid panel) are small, reaching only to 6-9\% near sunrise. The two model experiments with the ChemCam PWC (well-mixed: thick line, low moist layer: thin line) are on the low side of the REMS-H observations, whereas values from the increased-PWC experiment ( $11.5 \mu \mathrm{m}$, dotted line) are here rather good. RH from the no-adsorption experiment (dashed line) is clearly too high in the morning.

The REMS volume mixing ratios (bottom panel) decline during the late evening and night until sunrise, indicating some depleting process. The three simulations with adsorption produce values quite close to the observed 05-06LT minima of vmr, roughly covering the sol-to-sol range of 20-40 ppmv. The depleted layer of moisture is quite shallow, only 100-200 m (Savijärvi et al., 2016), so the column-PWC does not change notably during the sol. In the no-adsorption simulation (dashed) $\mathrm{vmr}$ is effectively constant during the sol, even though vertical diffusion of moisture is active both in the air and in the regolith. This leads to excessively high RH and vmr at nighttime. Hence the nocturnal diffusion into the soil is alone (i.e. without adsorption) not an effective remover of air moisture in the diurnal timescale.

The mixing ratios increase after sunrise (in the model due to desorption, upward diffusion and convection). The REMS-H values for vmr are unfortunately unreliable during daytime (because of very small, inaccurate $\mathrm{RH}$ ), but the ChemCam vmr (72 ppmv at 10:20 LT) provides here a kind of validation. ChemCam vmr estimates come from applying a well-mixed assumption to the ChemCam PWC (McConnochie et al., 2018), and they may actually be quite accurate near the surface, as will be discussed in Section 6. ChemCam vmr is in Figure 3 close to the well-mixed model's vmr curve (thick line), which grows to about $90 \mathrm{ppmv}$ in the afternoon. The experiment with a low moist layer (thin line) leads to slightly higher vmr, and the one with increased PWC (11.5 $\mu \mathrm{m}$, dotted) even higher, vmr then growing up to $140 \mathrm{ppmv}$ in the afternoon. Adsorption begins to strongly deplete the near-surface moisture from about 16LT onward in all the three simulations with adsorption.

Steele et al. (2017) presented mesoscale model simulations for Gale at around Ls $321^{\circ}$ within the warm season. The TES MY26 data-assimilated initial PWC was about $10 \mu \mathrm{m}$ over the Gale region. Their results with the J97 adsorption (fig. 24) indicate at the MSL site a surface vmr of about 40 ppmv at 06LT and about 100 ppmv at 16LT, not too different from the above warm season observations and column model results.

\section{Diurnal comparison during the cool season}

Figure 4 displays comparisons for Ls $90^{\circ}$ within the cool season, the ChemCam-observed local 
PWC being $5.31 \mu \mathrm{m}$ with $\tau 0.41$ (dust fraction 0.84 ) and p $8.44 \mathrm{mb}$ at Ls $89.93^{\circ}, 11: 43 \mathrm{LT}$, MY32. The REMS-H temperatures for three sols around Ls $90^{\circ}$ (top panel) are about 20K lower than at Ls $271^{\circ}$, with some sol-to-sol scatter. The model's T1.6m values match the nighttime and morning REMS-H observations again quite well but the afternoon T-maximum remains too cold. The three simulations with adsorption indicate here air frost points $\mathrm{T}_{\mathrm{f}}$ below the air and ground temperatures, so no frost nor fog is expected. However, in the no-adsorption simulation, $\mathrm{T}_{\mathrm{f}}$ does hit the surface temperature at around 02LT and ground frost is thereafter deposited until sunrise.

The observed nighttime maxima of relative humidity at 04-06LT are here in the range of 30-45\% with wide sol-to-sol scatter. Adsorptive simulations with ChemCam local PWC (thin and thick solid lines) again display RH on the low side of REMS-H observations, whereas that with increased PWC (dots) appears on the high side. The same holds for the nocturnal 01-07LT mixing ratios, which are now quite low (10-15 ppmv), displaying only little sol-to-sol scatter.

The ChemCam vmr of $56 \mathrm{ppmv}$ at 11:43LT is here located between the well-mixed and low moist layer simulation curves. Increased PWC $(9.3 \mu \mathrm{m}$, dotted) provides on the other hand daytime values of vmr, which are much higher, up to 95 ppmv. In the no-adsorption experiment (dashed), formation of ground frost depletes the near-surface vmr from 02LT onward. After sunrise rapid sublimation of frost returns the deposited moisture back to the air, increasing vmr temporarily at around 08LT near the surface.

In Steele et al. (2017) the cool season simulation (their fig. 28, Ls 69 ${ }^{\circ}$; PWC about $7 \mu \mathrm{m}$ over Gale) indicates surface-vmr of about $20 \mathrm{ppmv}$ at 06LT and $80 \mathrm{ppmv}$ at 16LT at the MSL site, again rather consistent with the above observations and column model results.

\section{The annual cycle}

Results from the annual cycle simulations are displayed in two parts for clarity. Figure 5 shows all the ChemCam daytime vmr values for the cool season $\left(\operatorname{Ls~} 0^{\circ}-135^{\circ}\right)$, together with the model's respective $1.6 \mathrm{~m}$ vmr at the ChemCam observation time, and at 05:00LT (the typical hour of minimum vmr). The two model daytime values are from the ChemCam PWC-forced simulations with adsorption, for the well-mixed and low moist layer assumptions. Simulations with increased PWC produced quite high vmr values (not shown), which are consistently about 50 ppmv above the ChemCam vmr, as was demonstrated in Figures 3-4.

In Figure 5 the lowest values of daytime ChemCam vmr occur at around Ls $30^{\circ}-70^{\circ}$, as discussed in McConnochie et al. (2018), and not during the coldest sols, which occur around Ls $90^{\circ}$ (Figure 2). The ChemCam vmr then increase toward Ls $135^{\circ}$. Well-mixed model's vmr values $(*)$ are typically slightly lower than the ChemCam vmr, whereas the low moist layer experiments $(+)$ tend to overestimate for higher vmr. At 05LT the model's vmr values are consistently quite low, due to depletion by adsorption in a shallow layer near the surface, as were the REMS-H observations in Figure 4.

Frost did not form in any of the simulations with adsorption, not even with increased PWC. However, when adsorption was switched off, frost did occur every night during Ls $86^{\circ}-142^{\circ}$. Frost has not been observed so far along the Curiosity track (Martinez et al., 2016, 2017), giving further support to the adsorption assumption for the nocturnal depletion.

Figure 6 similarly covers the warm season, Ls $135^{\circ}-360^{\circ}$. It is rather unfortunate that the probably most humid period at around Ls $160^{\circ}-190^{\circ}$ did not have any ChemCam sky scans. After about Ls $210^{\circ}$ the vmr values stay rather steady at $50-100 \mathrm{ppmv}$ with a weak decline toward Ls $360^{\circ}$. The 
well-mixed model's values of vmr at $1.6 \mathrm{~m}$ are here close to the ChemCam vmr, whereas the low moist layer experiments produce values, which are higher by 20-40 ppmv, and the increased PWC simulations (not shown), by about 50 ppmv.

The model's 05LT vmr values are consistently much lower than vmr at 10-13LT in Figures 5-6. The respective night depletion ratio $\mathrm{R}=\operatorname{vmr}(05 \mathrm{LT}) / \mathrm{vmr}$ (daytime) is plotted in Figure 7, using the ChemCam and model's 10-13LT vmr. It is seen that $\mathrm{R}$ is nearly constant and about 0.25 throughout the martian year, with lowest values during the cool season and highest values during the moist season. Excessive values of model-R (e.g. at Ls $82^{\circ}$ and at Ls $315^{\circ}$ ) may partly be due to the early ChemCam scans (around 10LT, Figure 2), when desorption has not yet been operating for long in the model. Hence the model-vmr at $1.6 \mathrm{~m}$ is about $20 \%$ lower at 10LT than it will be during midday (Figures 3-4), whereas the ChemCam vmr is based on the column-PWC, which does not display strong diurnal variation (Figure 1).

\section{Discussion}

In Figure 4 from the cool season there are considerable sol-to-sol variations at 00-05LT in the observed high and thus relatively accurate REMS-H relative humidities. They are anti-correlated with the respective sol-to-sol variations in the temperatures (e.g. at 00LT and 05LT). The low volume mixing ratios exhibit on the other hand only little sol-to-sol variation in this season in Figure 4 (and in Martinez et al., 2017), mainly because the anti-correlated variations of RH and T tend to compensate each other in the evaluation of vmr. One may thus suspect that the low nocturnal absolute humidity at Curiosity is much the same from sol to sol during the cool season. Therefore any sol-to-sol changes in temperatures (perhaps due to weak local advection effects) are immediately carried on to the relative humidities. This also implies that REMS-H is measuring air temperatures and relative humidities independently and fairly accurately during the night.

In contrast, during the warm season the observed hourly 01-06LT temperatures (and thus $\mathrm{e}_{\text {sat }}(\mathrm{T})$ ) are much the same from sol to sol, by Figure 3. Hence the large observed sol-to-sol variations in the high vmr during the warm season lead to relatively large sol-to-sol variations around the small RH, as seen in Figure 3 and in Martinez et al. (2017).

In general the REMS-H observations display a clear depletion of near-surface absolute moisture in the evening and night during all seasons along the Curiosity track, by some process or processes. This prevents frost. Our column model with adsorption is on the other hand close to the ChemCamobserved daytime vmr and to the pre-dawn much smaller REMS-H-observed vmr, whereas the noadsorption experiments produce too high vmr at night. Hence adsorption is likely at Gale throughout the year.

When adopting higher PWC mimicking the large-scale TES and CRISM retrievals for Gale (Mc Connochie et al., 2018), both our column model and the Steele et al. (2017) mesoscale model appear to produce higher but still reasonable RH and vmr at Curiosity during the night, but somewhat too high vmr during the day compared to the ChemCam and local CRISM vmr results. This may mean that the air within the crater is locally dry during daytime. On the other hand, if adsorption is switched off in these models, $\mathrm{RH}$ and vmr remain much too high during the night. Furthermore, this leads to formation of frost at the MSL site during the cool season, by both models. Frost has not been observed along the Curiosity track so far (Martinez et al., 2016, 2017).

We next discuss the vertical structure of moisture at Gale. Figure 8 displays the midday vmr profiles for the MSL site from MCD at Ls $90^{\circ}$ and $270^{\circ}$ (scaled by a constant factor $(0.38,0.54)$ at all heights to match the local ChemCam-observed PWC of Figures 3-4). Shown are also the respective 
'well-mixed' and 'low moist layer' vmr profiles. At Ls $270^{\circ}$ the GCM-based MCD indicates warmseason moisture well mixed to high altitudes, displaying a broad maximum at about $35 \mathrm{~km}$ height. In contrast, at $\mathrm{Ls} 90^{\circ}$ a sharp cool-season maximum of moisture is seen at about $10 \mathrm{~km}$ height, with rapid decay aloft. Most importantly, in both seasons the MCD profiles display relatively low values of vmr near the surface. These low values are due to Hadley circulation, which near the surface transports the dryness of the respective winter hemisphere to the equatorial latitudes (e.g. Fig. 11.18, Montmessin et al., 2017). According to MCD data the near-surface dryness dominates throughout the year at the MSL site, except at the peak of the moist season (which was not sampled by the present ChemCam sky scans).

Some interesting matters can be deduced from Figure 8. Assuming that the MCD profiles are close to truth, at Ls $270^{\circ}$ the well-mixed assumption is relatively valid for vmr. At the surface it gives an estimate just 6\% higher than MCD. On the other hand the low moist layer assumption is clearly no good during the warm season; at the surface it leads to a large overestimate of about $35 \%$. For Ls $90^{\circ}$ during the cool season, the low moist layer profile appears at first sight better in general than the well-mixed one. However, at the surface it does overestimate MCD by about $20 \%$, whereas the well-mixed profile is again quite good at the very surface, underestimating MCD there by only about $5 \%$.

Thus, due to the fortunate Hadley cell effect, the simple assumption of vertically fully well-mixed moisture manages to produce a fairly good estimate for midday surface-vmr at the MLS site (but note that this is only valid due to the equatorial latitude!). Therefore the ChemCam well-mixed vmr is probably not far from the true daytime surface vmr at Curiosity throughout most of the martian year. Our well-mixed model's daytime vmr $(*)$ is in turn fairly close to the ChemCam vmr (the filled circles) in Figures 5-6, especially during the warm season. In contrast, the low moist layer simulations (+) produce large overestimates to the ChemCam vmr during the warm season, being closer to them during the cool season. Hence our idealized modeling results and the REMS-H and ChemCam observations give support to the seasonally varying vertical distribution of moisture and a dry lower equatorial atmosphere as predicted by the GCM experiments.

\section{Conclusion}

The local column precipitable water contents from MSL ChemCam passive sky scan retrievals (McConnochie et al., 2018) were used to force a local atmosphere-subsurface column model. The 113 scans covered more than a martian year. ChemCam estimates for the water vapor volume mixing ratio (assuming well-mixed moisture) and REMS-H hourly observations of air temperature, relative humidity and vmr at $1.6 \mathrm{~m}$ height were compared with model results. The sol-to-sol PWCconserving porosity of the regolith turned out to be about $30 \%$ in the present model framework.

The REMS-H observations point to a decrease of vmr (i.e. depletion of near-surface water vapor) every evening and night throughout the year. The same was observed at the polar Phoenix site in summer (Zent et al., 2016), suggesting a global process. If adsorption is allowed, the model's predawn results are quite similar to REMS-H values of T, RH and vmr throughout the year. The indicated night depletion ratio is about 0.25 at all seasons. If adsorption is not allowed, $\mathrm{RH}$ and vmr become excessive during every night at all seasons compared to the REMS-H values, leading to surface frost every night between Ls $82^{\circ}-146^{\circ}$. Frost has not been observed. As brine formation has been shown to be unlikely along the Curiosity track, adsorption hence appears to be the main depleting process.

The REMS-H vmr values are unfortunately unreliable during the day but the daytime ChemCam vmr estimates are available. They appear to be close to the midday surface-vmr from the GCM- 
based Mars Climate Database profiles for the MLS site (scaled to the ChemCam PWC) during all seasons. The well-mixed model's daytime near-surface vmr is in turn close to the respective ChemCam vmr throughout the year, whereas simulations with a low moist layer $(15 \mathrm{~km})$ lead to large overestimates during the warm season, being nearer to the ChemCam vmr during the cool season. Higher TES/CRISM-like regional PWC leads to even larger daytime overestimates throughout the year, although the nighttime values for RH and vmr remain reasonable. Hence the crater appears drier at daytime than the surrounding areas, and the results support a seasonally varying vertical distribution of moisture with a dry lower atmosphere, as suggested by GCM experiments.

Acknowledgements: The work was supported by the Academy of Finland grants 131723, 132825 and 310509. We thank Pierre-Yves Meslin and an anonymous reviewer for good comments, which helped to improve the article.

\section{References:}

Fischer E, Martinez GM, Renno NO, 2018. The Phoenix Lander's relative humidity sensor recalibration: new results and analysis. $49^{\text {th }}$ Lunar and Planetary Science Conference 2018, LPI Contrib. No. 2083.

Gómez-Elvira GJ et al., 2012. REMS: The Environmental Sensor Suite for the Mars Science Laboratory Rover. Space Sci. Rev. 170, 583-640, doi:10.1007/s11214-012-9921-1.

Harri AM, et al., 2014a. Mars Science Laboratory relative humidity observations: initial results. J. Geoph. Res. Planets 119, 2132-2147, doi: 10.1002/2013JE004514

Harri AM, et al., 2014b. Pressure observations by the Curiosity rover: initial results. J.Geophys.Res. Planets 119, 82-92. doi: 10.1002/2013JE004423.

Hudson TL, Aharonson O, Schorghofer N, Farmer CB, Hecht MH, Bridges NT, 2007. Water vapor diffusion in Mars subsurface environments. J.Geophys.Res. 112, E05016.

Jakosky BM, Zent AP, Zurek RW, 1997. The Mars water cycle: Determining the role of exchange with the regolith. Icarus 130, 87-95.

Martínez GM, Fischer E, Rennó NO, Sebastián E, Kemppinen O, Bridges N, Borlina CS, Meslin PY, Genzer M, Harri AM, Vicente-Retortillo A, 2016. Likely frost events at Gale crater: analysis from MSL/REMS measurements. Icarus 280, 93-102.

Martínez GM, Newman CN, De Vicente-Retortillo A, Fischer E, Renno NO, Richardson MI, Fairén AG, Genzer M, Guzewich SD, Haberle RM, Harri AM, 2017. The Modern Near-Surface Martian Climate: A Review of In-situ Meteorological Data from Viking to Curiosity. Space Sci. Rev. pp.144.

Martin-Torres FJ, et al., 2015. Transient liquid water and water activity at Gale crater on Mars. Nat. Geosci. 8, 357-361, doi:10.1038/NGEO2412.

McConnochie TH, et al., 2018. Retrieval of water vapor column abundance and aerosol properties from ChemCam passive sky spectroscopy. Icarus 307, 294-326, doi:10.1016/j.icarus.2017.10.043. 
Millour E et al., 2015. The Mars Climate Database (MCD version 5.2). EPSC2015-438.

Montmessin F, Smith MD, Langevin Y, Mellon MT, Fedorova A, 2017. The water cycle, pp 338373. In "The Atmosphere and Climate of Mars", Haberle RM, Todd Clancy R, Forget F, Smith MD, Zurek RW, eds. Cambridge University Press, 644 pp.

Navarro T, et al., 2014. Global climate modeling of the Martian water cycle with improved microphysics and radiatively active water ice clouds. J.Geophys.Res.Planets 119, 1479-1495.

Richardson MI, Wilson RJ, 2002. Investigation of the nature and stability of the martian seasonal water cycle with a general circulation model. J.Geophys.Res. (Planets) 107. doi:10.1029/2001JE001536. 7-1.

Rivera-Valentin EG, Gough RV, Chevrier VF, Primm KM, Martinez GM, Tolbert M, 2018. Constraining the potential liquid water environment at Gale crater, Mars. J.Geophys.Res. (Planets), doi:10.1002/2018JE005558.

Savijärvi H, Määttänen A, 2010. Boundary-layer simulations for the Mars Phoenix lander site. Quart. J. Roy. Meteor. Soc. 136, 1497-1505, doi:10.1002/qj.650

Savijärvi H, Harri AM, Kemppinen O, 2015. Mars Science Laboratory diurnal moisture observations and column integrations. J.Geophys.Res. Planets 120, 1011-1021, doi: 10.1002/2014JE004732

Savijärvi H, Harri AM, Kemppinen O, 2016. The diurnal water cycle at Curiosity: role of exchange with the regolith. Icarus 265, 63-69, doi:10.1016/j.icarus.2015.10.00

Savijärvi H, Paton M, Harri AM, 2017: New column simulations for the Viking landers: winds, fog, frost, adsorption? Icarus, doi: 10.1016/j.icarus.2017.11.007.

Steele LJ, Lewis SR, Patel MR, Montmessin F, Forget F, Smith MD, 2015. The seasonal cycle of water vapor on Mars from assimilation of thermal emission spectrometer data. Icarus 237, 97-115.

Steele LJ, Balme MR, Lewis SR, Spiga A, 2017. The water cycle and regolith-atmosphere interaction at Gale crater, Mars. Icarus 280, 56-79.

Toigo AD, Smith MD, Seelos FP, Murchie SL, 2013. High spatial and temporal resolution sampling of Martian gas abundances from CRISM spectra. J.Geophys.Res., Planets 118, 89, doi: 10.1029/2012JE004147.

Zent AP, Hecht MH, Hudson TL, Wood SE, Chevrier VF, 2016. A revised calibration function and results for the Phoenix mission TECP relative humidity sensor. J. Geophys. Res. (Planets) 121, 626651. 
508

509

510

511

512

513

514

515

516

517

518

519

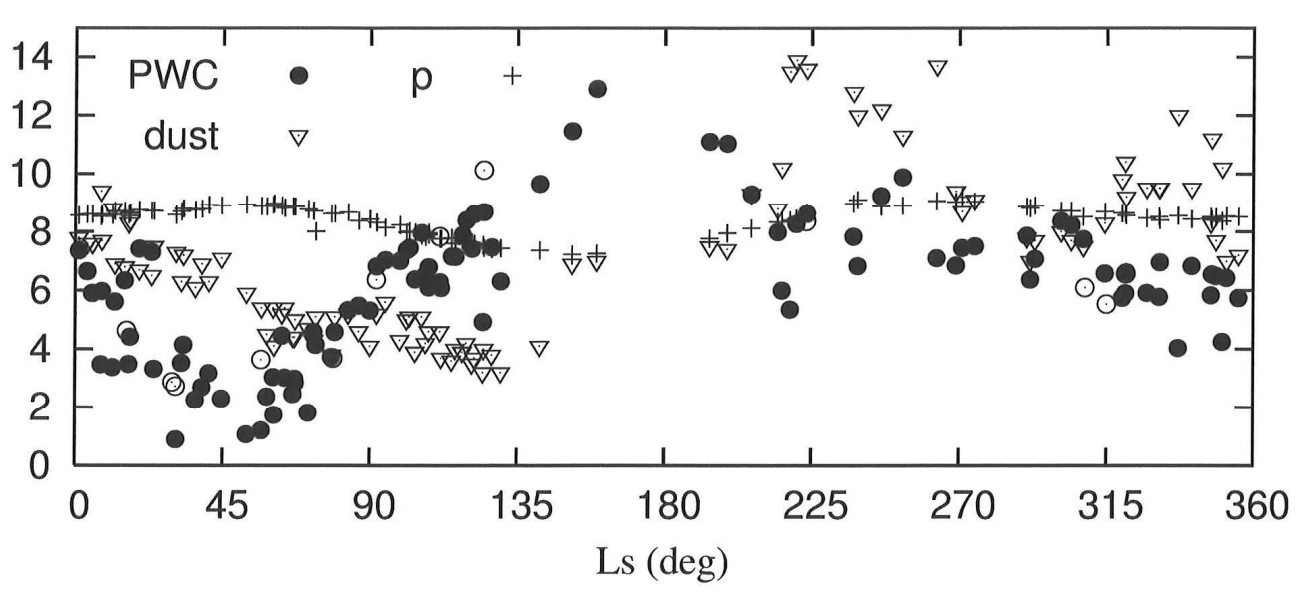

Figure 1. Column precipitable water content (PWC, in $\mu \mathrm{m}$ ) from MSL ChemCam passive sky scans (filled circles from daytime, open circles from morning), column total opacity, mostly by dust ( $\tau$ multiplied by 10 ) from MSL MastCam, and surface pressure ( $p$, in mb) from MSL REMS-P.

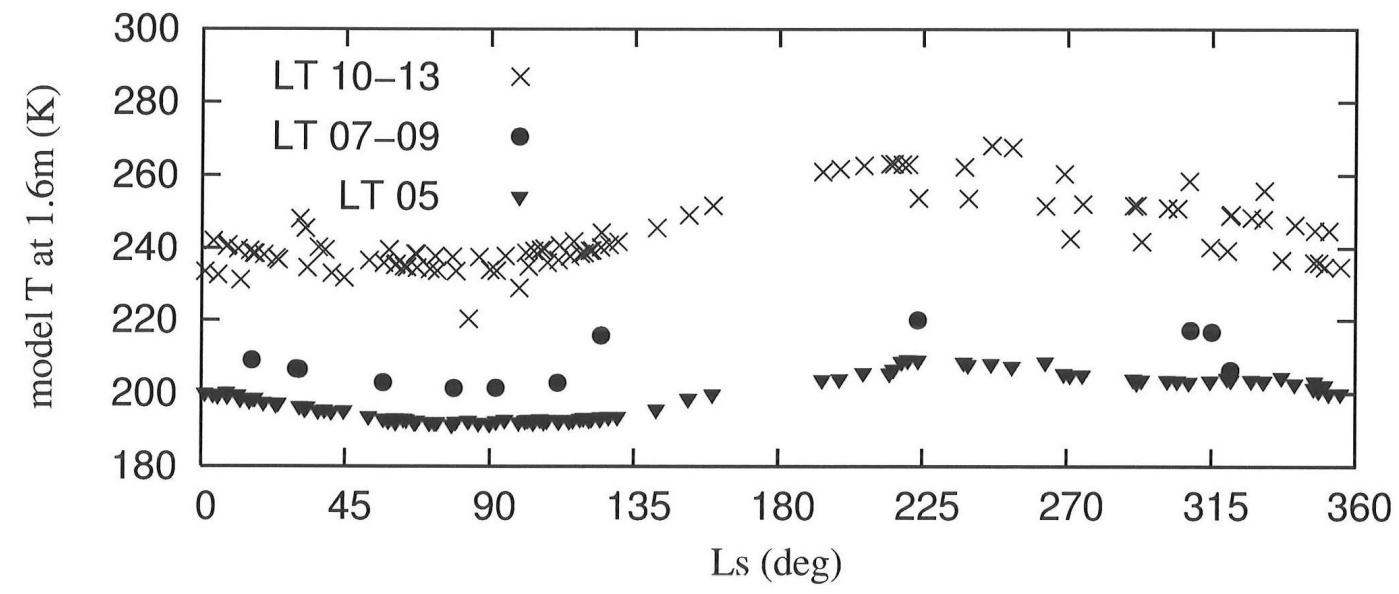

Figure 2. Model 1.6 m temperatures at 05:00LT and at the nearest hour (e.g. 11:00LT) for each ChemCam PWC observation of Figure 1. 

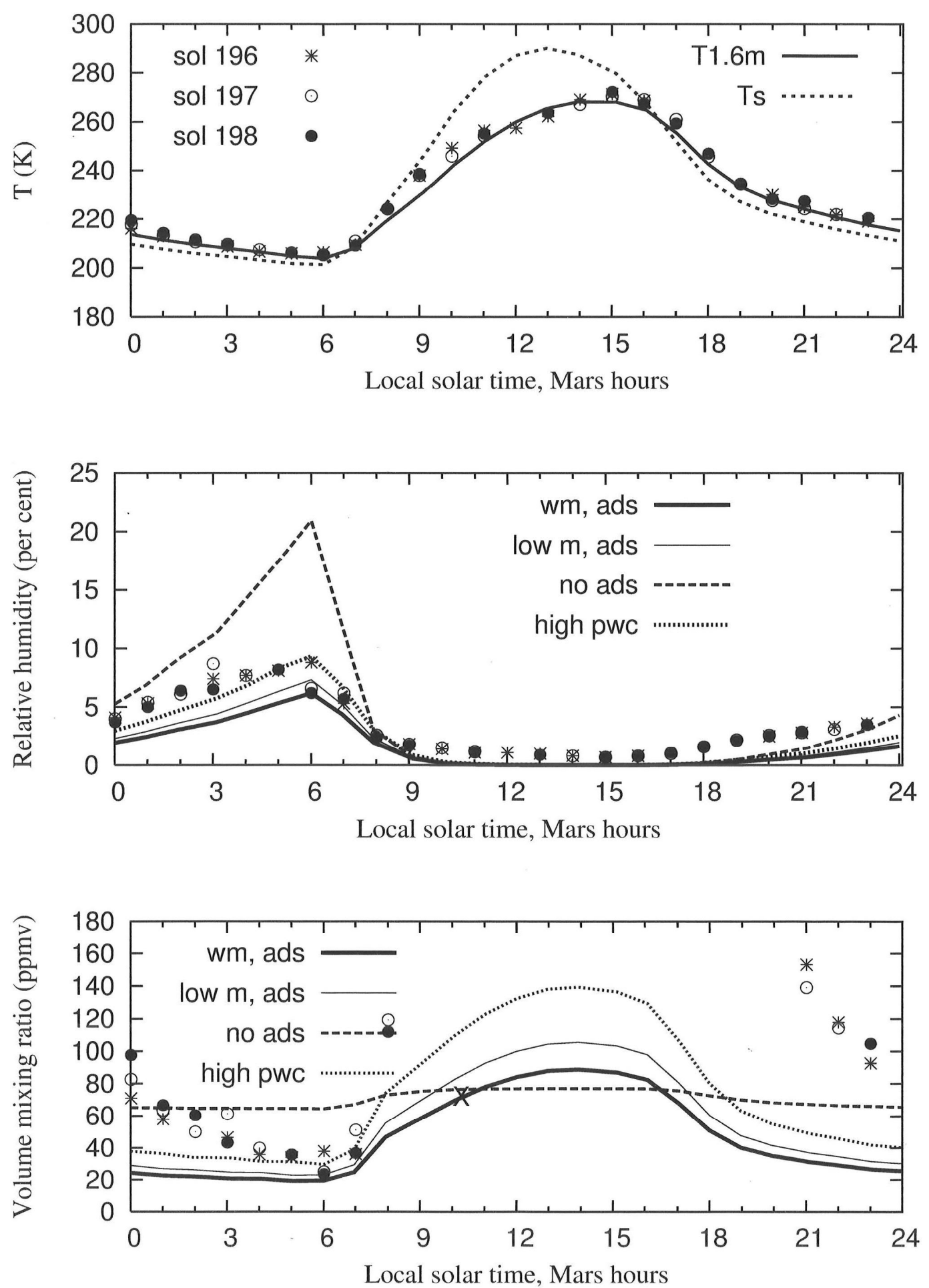

Figure 3. Hourly REMS-H observations (marks) for three sols at around Ls $271^{\circ}$ during the warm season, and model results in the four experiments (lines). Top panel: T1.6 m and Ts. Mid panel: Relative humidity at $1.6 \mathrm{~m}$. Bottom panel: Volume mixing ratio at $1.6 \mathrm{~m} ; \mathrm{X}$ is the ChemCam vmr 

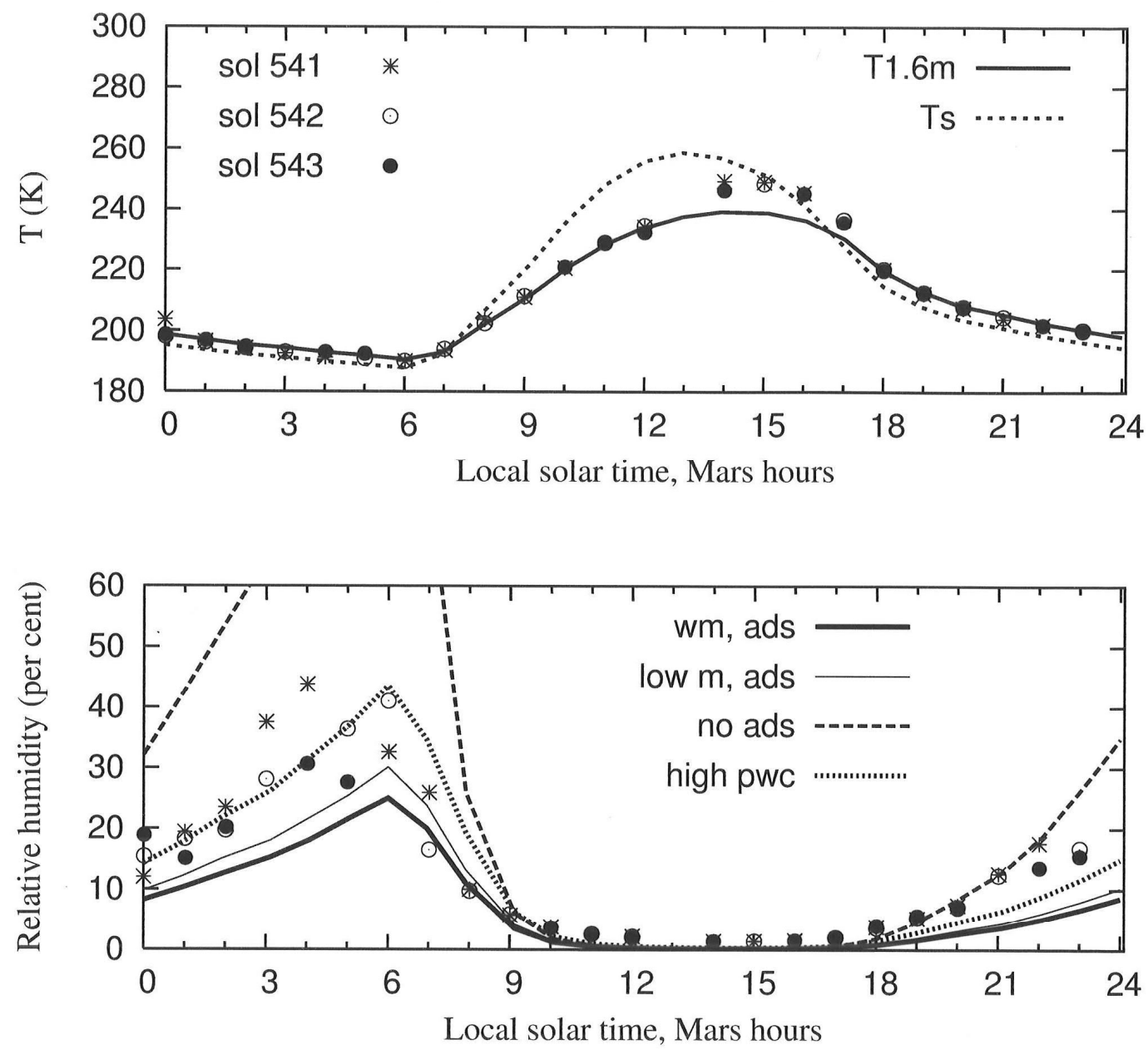

Figure 4. As Figure 3 but for the cool season, at around Ls $90^{\circ}$.

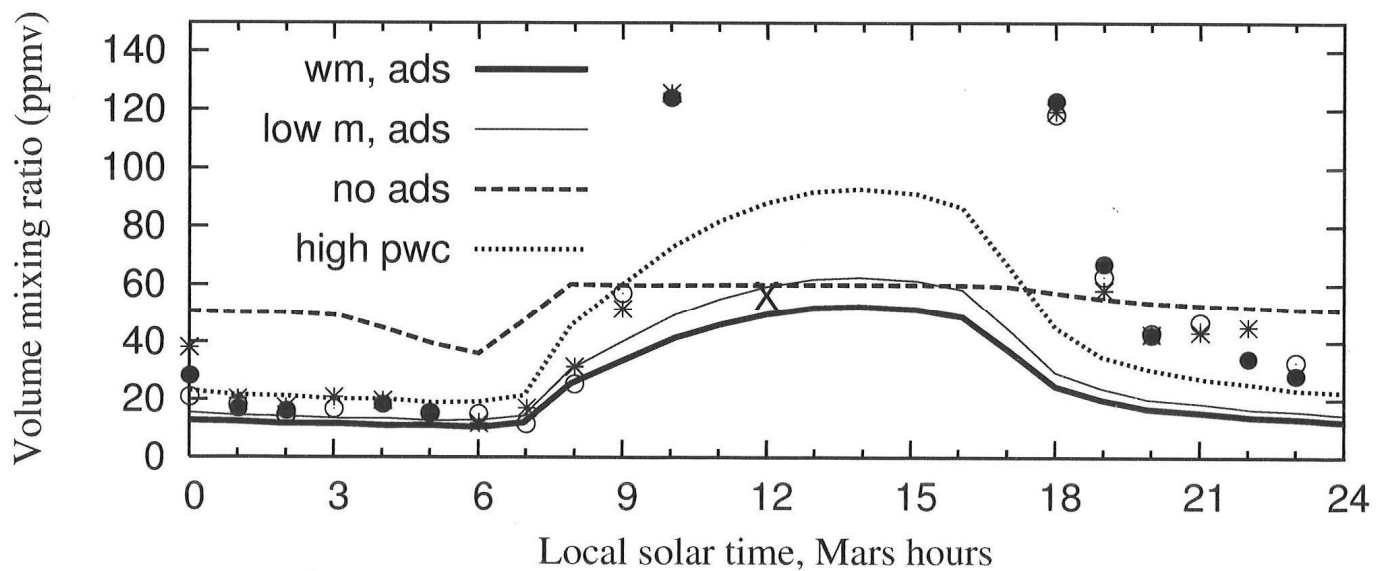




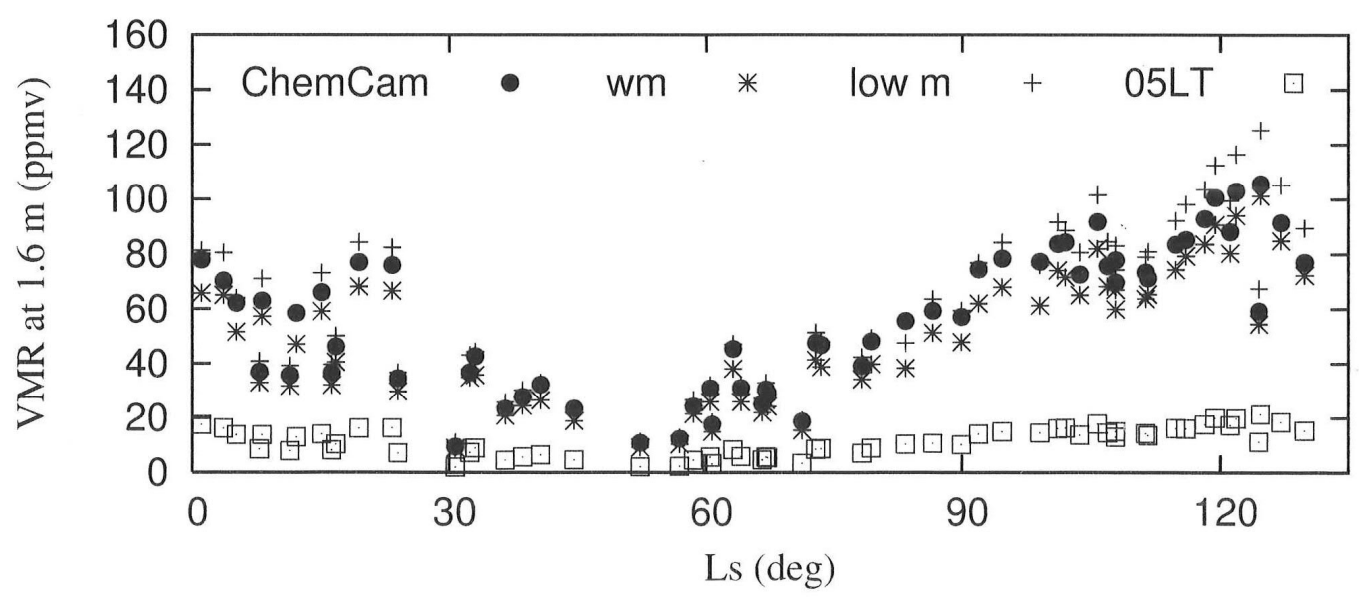

Figure 5. The cool season (Ls $\left.0^{\circ}-135^{\circ}\right)$ volume mixing ratios from all ChemCam daytime scans (filled circles); model $1.6 \mathrm{~m}$ vmr at the scan time (well-mixed simulation *, low moist layer simulation + ), and at 05LT (squares). The Ls $90^{\circ}$ case was shown in Figure 4.

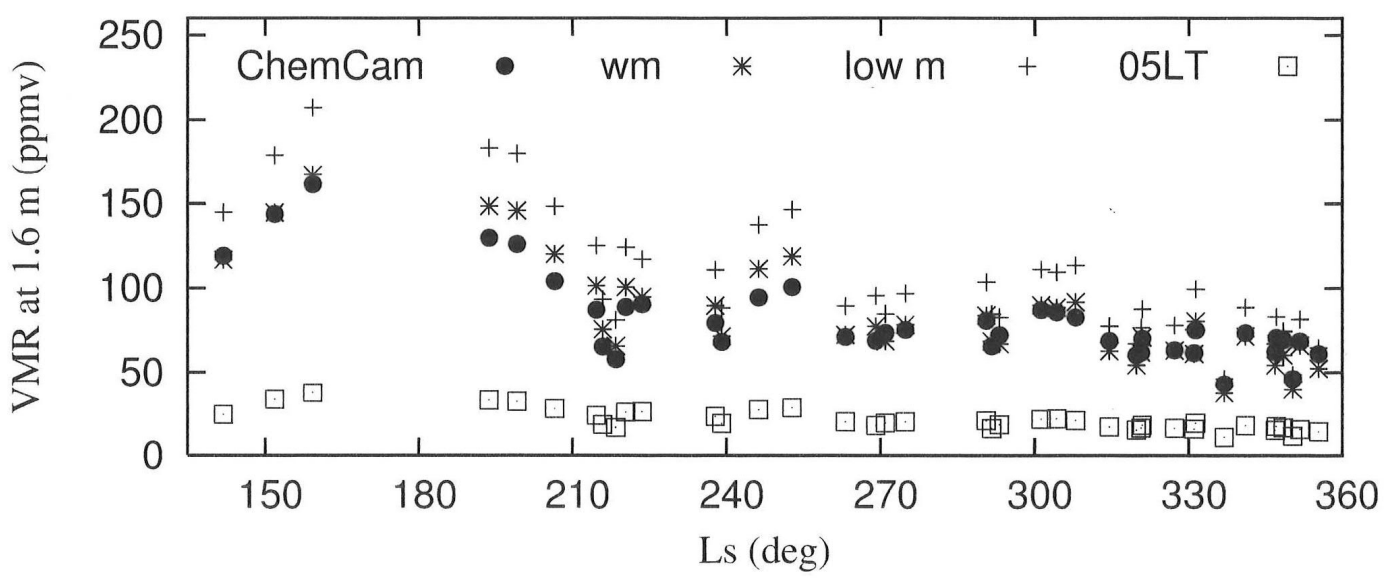

Figure 6. As Figure 5 but for the moist and warm seasons, Ls $135^{\circ}-360^{\circ}$. The Ls $271^{\circ}$ case was shown in Figure 3. 


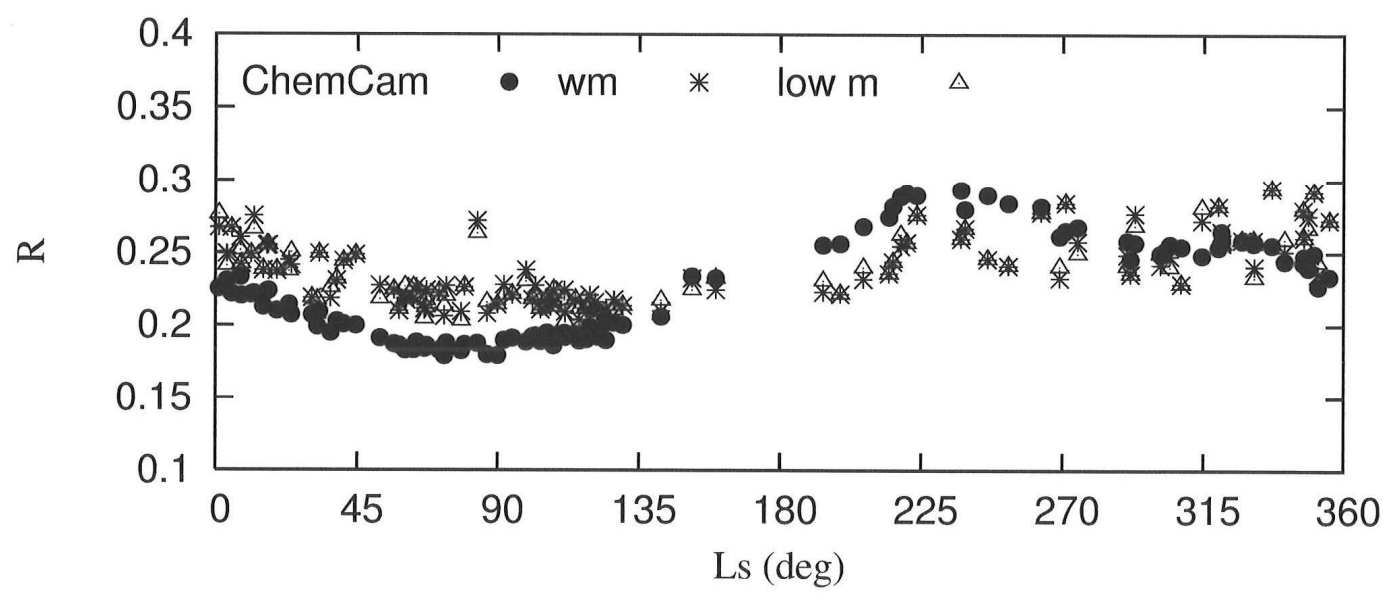

Figure 7. The night depletion ratio $\mathrm{R}(\mathrm{vmr}(05 \mathrm{LT}) / \mathrm{vmr}$ (daytime)) using the ChemCam vmr and the model $1.6 \mathrm{~m}$ vmr from Figures 5-6 (well mixed simulation: stars, low moisture layer simulation: triangles). The $05 \mathrm{LT}$ value for ChemCam $\mathrm{R}$ is from the well-mixed simulation.

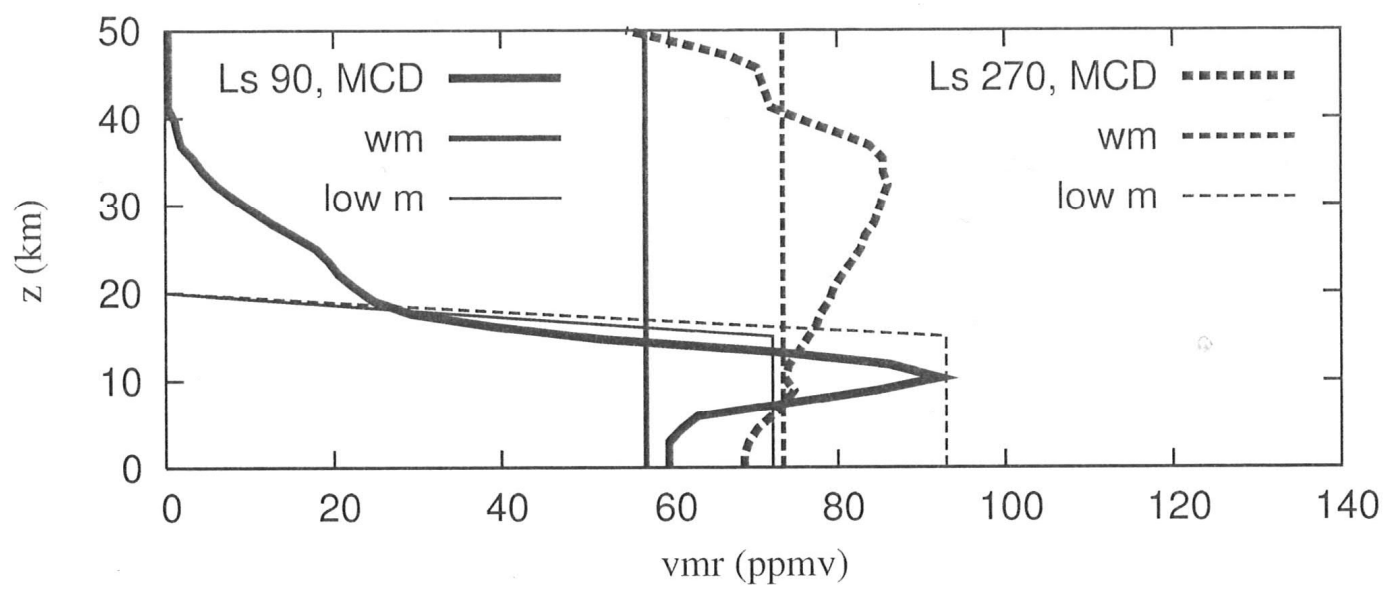

Figure 8. 12LT volume mixing ratio profiles (scaled to ChemCam PWC) at the MSL site for Ls $90^{\circ}$ and Ls $270^{\circ}$ from the GCM-based Mars Climate Database (MCD), and the respective same-PWC profiles for the well-mixed (wm) and low moist layer (low m) assumptions. 